\title{
Bosentan added to sildenafil therapy in patients with pulmonary arterial hypertension
}

\author{
Vallerie McLaughlin ${ }^{1}$, Richard N. Channick ${ }^{2}$, Hossein-Ardeschir Ghofrani ${ }^{3}$, \\ Jean-Christophe Lemarié ${ }^{4}$, Robert Naeije ${ }^{5}$, Milton Packer ${ }^{6}$, Rogério Souza ${ }^{7}$, \\ Victor F. Tapson ${ }^{8}$, Jonathan Tolson ${ }^{9}$, Hikmet Al Hiti ${ }^{10}$, Gisela Meyer ${ }^{11}$ and \\ Marius M. Hoeper ${ }^{12}$
}

Affiliations: ${ }^{1}$ Department of Internal Medicine, Division of Cardiovascular Medicine, University of Michigan,
Ann Arbor, MI, USA. ${ }^{2}$ Pulmonary and Critical Care, Massachusetts General Hospital, Boston, MA, USA.
${ }^{3}$ University of Giessen and Marburg Lung Center, Giessen, Germany. ${ }^{4}$ Department of Statistics, Effi-Stat,
Paris, France. ${ }^{5}$ Department of Cardiology, Erasme University Hospital, Brussels, Belgium. ${ }^{\circ}$ Department of
Clinical Sciences, University of Texas Southwestern Medical Center, Dallas, TX, USA. ${ }^{7}$ Pulmonary Department,
Heart Institute, University of São Paulo Medical School, São Paulo, Brazil. ${ }^{8}$ Division of Pulmonary and Critical
Care Medicine, Cedars-Sinai Medical Center, ${ }^{10}$ Los Angeles, CA, USA. ${ }^{9}$ Global Medical Affairs, Actelion
Pharmaceuticals Ltd, Allschwil, Switzerland. ${ }^{10}$ Department of Cardiology, IKEM, Prague, Czech Republic.
${ }^{11}$ Complexo Hospitalar Santa Casa De Porto Alegre, Pulmonary Vascular Research Institute, Porto Alegre,
Brazil. ${ }^{12}$ Department of Respiratory Medicine, Hannover Medical School and German Center of Lung Research
(DZLI, Hannover, Germany.

Correspondence: Vallerie McLaughlin, Department of Internal Medicine, Division of Cardiovascular Medicine, University of Michigan Health System, Ann Arbor, MI 48109-5853, USA. E-mail: vmclaughهumich.edu

ABSTRACT The safety and efficacy of adding bosentan to sildenafil in pulmonary arterial hypertension $(\mathrm{PAH})$ patients was investigated.

In this prospective, double-blind, event-driven trial, symptomatic $\mathrm{PAH}$ patients receiving stable sildenafil ( $\geqslant 20 \mathrm{mg}$ three times daily) for $\geqslant 3$ months were randomised (1:1) to placebo or bosentan (125 mg twice daily). The composite primary end-point was the time to the first morbidity/mortality event, defined as all-cause death, hospitalisation for $\mathrm{PAH}$ worsening or intravenous prostanoid initiation, atrial septostomy, lung transplant, or PAH worsening. Secondary/exploratory end-points included change in 6-min walk distance and World Health Organization functional class at 16 weeks, change in N-terminal pro-brain natriuretic peptide (NT-proBNP) over time, and all-cause death.

Overall, $334 \mathrm{PAH}$ patients were randomised to placebo $(n=175)$ or bosentan $(n=159)$. A primary endpoint event occurred in $51.4 \%$ of patients randomised to placebo and $42.8 \%$ to bosentan (hazard ratio 0.83 , 97.31\% CI 0.58-1.19; $\mathrm{p}=0.2508$ ). The mean between-treatment difference in 6-min walk distance at 16 weeks was $+21.8 \mathrm{~m}$ (95\% CI +5.9-37.8 m; p=0.0106). Except for NT-proBNP, no difference was observed for any other end-point. The safety profile of bosentan added to sildenafil was consistent with the known bosentan safety profile.

In COMPASS-2, adding bosentan to stable sildenafil therapy was not superior to sildenafil monotherapy in delaying the time to the first morbidity/mortality event.

@ERSpublications

COMPASS-2: adding bosentan was not superior to sildenafil alone in delaying time to first morbidity/mortality event http://ow.ly/NiM65

For editorial comments see Eur Respir J 2015; 46: 297-298 [DOI:10.1183/13993003.00907-2015]

This article has supplementary material available from erj.ersjournals.com

Received: Nov 042014 | Accepted after revision: April 30 2015 | First published online: June 252015

Clinical trial: This study is registered at www.clinicaltrials.gov with identifier number NCT00303459.

Support statement: The COMPASS-2 study was funded by Actelion Pharmaceuticals Ltd. Funding information for this article has been deposited with FundRef.

Conflict of interest: Disclosures can be found alongside the online version of this article at erj.ersjournals.com

Copyright @ERS 2015 


\section{Introduction}

Pulmonary arterial hypertension (PAH) is a severe and progressive disease leading to right heart failure and death. Three major mechanistic pathways, the endothelin, prostacyclin and nitric oxide pathways, are known to be involved in the pathogenesis of PAH and therapies that target these pathways are available [1-3]. Combining agents that target more than one pathway is an attractive option for the treatment of $\mathrm{PAH}$ and may offer additional long-term benefits compared with monotherapy $[4,5]$. The proceedings from the third World Symposium on Pulmonary Hypertension state that, despite the lack of supporting trial data, combination therapy could be considered an option for patients deteriorating on monotherapy [6]. In addition to the need for controlled data on the efficacy and safety of combination therapy in patients with $\mathrm{PAH}$, it was also recognised at the time of the third World Symposium on Pulmonary Hypertension that future trial design would see a shift from short- to long-term studies and that composite primary end-points indicative of disease progression would be more useful than other end-points typically used in clinical trials at that time [7].

COMPASS-2 was designed as an event-driven study to evaluate the effect of bosentan on the long-term clinical course of patients with symptomatic PAH already receiving sildenafil, using a composite primary end-point of time to first confirmed morbidity/mortality event.

\section{Methods}

\section{Study oversight}

The steering committee designed and oversaw the conduct of the trial and data analysis in collaboration with the sponsor (Actelion Pharmaceuticals Ltd, Allschwil, Switzerland). The trial was conducted in compliance with good clinical practice and the principles of the Declaration of Helsinki, was approved by local institutional review boards or independent ethics committees, and was monitored by an independent data monitoring committee. Data were collected, managed and analysed by the sponsor according to a predefined statistical analysis plan. The manuscript was prepared by the authors, who had unrestricted access to the data, agreed to submit the manuscript for publication, and assume responsibility for the accuracy and completeness of the data and analyses.

\section{Patients}

Patients were $\geqslant 18$ years old with symptomatic PAH confirmed by right heart catheterisation and included patients with idiopathic $\mathrm{PAH}$, hereditary $\mathrm{PAH}$, or $\mathrm{PAH}$ associated with connective tissue disease, repaired congenital heart defects ( $\geqslant 2$ years repaired) or drugs and toxins. Patients were required to have a baseline 6-min walk distance (6MWD) of $150-480 \mathrm{~m}$ and to be receiving a stable dose of sildenafil $\geqslant 20 \mathrm{mg}$ three times daily for at least 3 months prior to randomisation. PAH medications other than sildenafil could not be used within 3 months prior to randomisation (supplementary table 1). Written informed consent was obtained from all patients.

\section{Study design and procedures}

COMPASS-2 was a prospective, international, randomised, double-blind, placebo-controlled, event-driven trial (www.clinicaltrials.gov identifier number NCT00303459). Patients fulfilling the entry criteria were randomised, in a 1:1 ratio, to receive placebo or bosentan, initially at $62.5 \mathrm{mg}$ twice daily for 4 weeks and then $125 \mathrm{mg}$ twice daily, while sildenafil therapy was continued at an unchanged dose. Patients who could not tolerate the study medication at $125 \mathrm{mg}$ twice daily could be down-titrated to $62.5 \mathrm{mg}$ twice daily at any time. After 16 weeks, the dose of sildenafil could be adjusted or discontinued, or patients could be switched to tadalafil.

Clinical assessments, including 6-min walk test, World Health Organization (WHO) functional class and a seven-point patient global self-assessment (PGSA) scale for PAH symptoms (supplementary file 1), were performed every 4 months following randomisation until the end of the study; these assessments were also performed if the patient experienced worsening PAH. Adverse events (AEs) and serious adverse events (SAEs) were recorded while patients were taking the study medication, and up to 1 and 28 days after permanent discontinuation of study drug for an AE or SAE, respectively. Liver function tests and haemoglobin levels were measured monthly while patients were receiving double-blind treatment. Double-blind treatment could be discontinued for safety reasons or if the patient started open-label treatment with an endothelin receptor antagonist following the occurrence of a primary end-point event. The study was stopped when the target number of primary end-point events was reached.

\section{Outcome measures}

The composite primary efficacy end-point was time to the first morbidity/mortality event, defined as time to death from any cause, hospitalisation for worsening PAH or start of intravenous prostanoid therapy, atrial septostomy, lung transplant, or worsening PAH. Worsening PAH was defined as: 1) moderate or marked worsening of PAH symptoms on the PGSA together with the initiation of a subcutaneous or 
inhaled prostanoid or use of open-label bosentan; or 2) no change or mild worsening of PAH symptoms accompanied by a decrease in $6 \mathrm{MWD}$ by more than $20 \%$ from the previous visit or by more than $30 \%$ from the baseline visit, together with the initiation of a subcutaneous or inhaled prostanoid or use of open-label bosentan. Patients reporting improvement in the PGSA were not considered to have experienced a worsening PAH event. All morbidity/mortality events were adjudicated in a blinded fashion by a clinical end-point committee and only confirmed first events were used in the analysis of the primary end-point.

The pre-specified secondary end-points were: 1) change in 6MWD at 16 weeks; 2) change in WHO functional class at 16 weeks; 3) time to the first occurrence of death from any cause, hospitalisation for $\mathrm{PAH}$ or start of intravenous prostanoid therapy, atrial septostomy, or lung transplant; and 4) death from any cause. Exploratory end-points included the change in N-terminal pro-brain natriuretic peptide (NT-proBNP).

\section{Statistical analysis}

The initial assumptions for the primary end-point were an annual rate of $21 \%$ on placebo with a risk reduced by $36 \%$ (hazard ratio (HR) 0.64 ) with bosentan and a negligible annual attrition rate. In addition, it was planned to conduct a single final analysis at 0.04 (two-sided), taking into account the existence of a co-primary end-point (change in 6MWD at 16 weeks) planned to be tested at 0.01 (two-sided). Over the course of the study, a number of amendments were introduced based on the evolution of knowledge in the field of $\mathrm{PAH}$, as well as the rate of enrolment and blinded evaluation of the overall event rate. On implementation of an amendment in 2007, the 6MWD end-point was changed from a co-primary end-point to a secondary endpoint and the Type I error associated with the single remaining primary end-point was increased to 0.05 (two-sided). On implementation of the final amendment in 2010, the assumptions were that the annual rate of a primary end-point event would be $20 \%$ in the placebo group, that this risk would be reduced by $43 \%$ (HR 0.57) with bosentan, that the annual attrition rate would be $5.7 \%$, and that two interim efficacy analyses would be performed at $50 \%$ and $75 \%$ of the target number of events. With an overall study-wise Type I error $(\alpha)$ set to 0.05 (two-sided) based on the log-rank test, when adjusted for two pre-planned blinded interim analyses at $50 \%$ and $75 \%$ of the target number of primary end-point events, the $\alpha$ for the final analysis of the primary end-point was 0.0269 and, thus, $97.31 \%$ confidence intervals were used in reporting the HRs. A total of 156 events were required to detect the pre-specified difference between the two groups with a power of $90 \%$.

The primary analysis was based on data from all randomised patients following the intention-to-treat principle. All time-to-event end-point analyses were displayed using Kaplan-Meier plots and the difference between the groups was tested for significance using the log-rank test. The proportion of patients with missing data for the primary end-point was calculated in patient-years and expressed as a percentage of the total information expected if all patients having not experienced a primary end-point event would have completed the study as planned. Pre-specified subgroup analyses were performed for the primary end-point; interaction tests were performed without correction for multiple testing and evaluated for significance with a threshold of $\mathrm{p}=0.10$. Change in $6 \mathrm{MWD}$ at 16 weeks was analysed with the Wilcoxon rank-sum test and the proportion of patients with an improved WHO functional class at 16 weeks was analysed with the Fisher's exact test. Missing assessments of 6MWD at 16 weeks and WHO functional class at 16 weeks were imputed with the use of either the last-observation-carried-forward method for patients who did not experience a primary end-point event within the first 16 weeks or a method that imputed data according to a worst-case scenario for patients who experienced a primary end-point event within the first 16 weeks (supplementary table 2). Repeated-measures analyses with the baseline value as a covariate were carried out for changes in NT-proBNP using mixed model techniques; assessments considered were those where $\geqslant 60 \%$ patients with NT-proBNP sampling performed had available values.

\section{Results}

\section{Patient disposition and duration of follow-up}

Between May 2006 and June 2012, 334 patients were enrolled at 83 centres in 13 countries: 175 were randomised to placebo and 159 to bosentan (figure 1). Baseline characteristics were balanced between the two groups (table 1). The population was predominantly female, with a mean age of 53.9 years, mean body mass index (BMI) of $28.8 \mathrm{~kg} \cdot \mathrm{m}^{-2}$ and mean time from diagnosis of 25.7 months, and predominantly WHO functional class III and of idiopathic aetiology (63.8\% of patients). The mean \pm SD duration of follow-up in the study was $39.7 \pm 22.6$ months for the placebo group and $38.0 \pm 21.9$ months for the bosentan group; $>70 \%$ of patients in both treatment groups were followed for $\geqslant 24$ months. The median daily dose of sildenafil during the study was $60.0 \mathrm{mg}$ for patients in the placebo and bosentan groups. Overall, 172 patients (placebo: 89 (50.9\%); bosentan: 83 (52.2\%)) did not complete the study: 77 patients died (placebo: 44; bosentan: 33) and 95 patients prematurely discontinued (placebo: 45; bosentan: 50), 58 of whom withdrew consent (placebo: 26; bosentan: 32) (figure 1). Of the 172 patients who did not complete the study, 63 did 
not experience a primary end-point event, resulting in $20 \%$ missing information for the primary end-point and $22 \%$ for the secondary end-point of time to death from any cause.

\section{Primary end-point}

A primary end-point event occurred in 90 (51.4\%) out of 175 patients and $68(42.8 \%)$ out of 159 patients in the placebo and bosentan groups, respectively (table 2). The HR for the primary end-point in the bosentan group, as compared with the placebo group, was 0.83 (97.31\% CI $0.58-1.19, p=0.2508$ ) (figure 2). Approximately half of the primary end-point events were categorised as "worsening PAH" (placebo: $52(57.8 \%)$ out of 90; bosentan: $32(47.0 \%)$ out of 68$)$. The overall treatment effect was consistent across all but two pre-specified groups; there were nominally significant interactions with 6MWD and geographical region (figure 3$)$. The HR for the primary endpoint in the per-protocol set $(n=270)$ was 0.88 (97.31\% CI 0.60-1.29; $\mathrm{p}=0.4601)$.

\section{Secondary and exploratory end-points}

As the primary objective of the study was not met, all other efficacy analyses are considered exploratory. The mean 6MWD worsened by $14.6 \pm 80.4 \mathrm{~m}$ in the placebo group and improved at 16 weeks by $7.2 \pm 66.0 \mathrm{~m}$ in the bosentan group; the difference between the groups $(21.8 \mathrm{~m}, 95 \%$ CI $5.9-37.8 \mathrm{~m})$ was nominally

\section{TABLE 1 Baseline characteristics in placebo and bosentan groups}

\begin{tabular}{|c|c|c|c|}
\hline & Placebo & Bosentan & All \\
\hline Patients $\mathrm{n}$ & 175 & 159 & 334 \\
\hline \multicolumn{4}{|l|}{ Sex } \\
\hline Female & $128(73.1)$ & $125(78.6)$ & $253(75.7)$ \\
\hline Male & $47(26.9)$ & $34(21.4)$ & $81(24.3)$ \\
\hline \multicolumn{4}{|l|}{ Age years } \\
\hline Mean \pm SD & $54.7 \pm 15.7$ & $52.9 \pm 15.4$ & $53.9 \pm 15.6$ \\
\hline Median (interquartile range) & $56.0(41.0-68.0)$ & $55.0(40.0-65.0)$ & $55.0(40.0-66.0)$ \\
\hline \multicolumn{4}{|l|}{$\mathrm{BMI}^{\#} \mathrm{~kg} \cdot \mathrm{m}^{-2}$} \\
\hline Mean \pm SD & $29.1 \pm 6.95$ & $28.5 \pm 6.83$ & $28.8 \pm 6.9$ \\
\hline Median (interquartile range) & $28.2(24.5-32.8)$ & $27.5(23.9-32.2)$ & $28.0(24.1-32.8)$ \\
\hline \multicolumn{4}{|l|}{ Geographical region } \\
\hline USA & $83(47.4)$ & 73 (45.9) & $156(46.7)$ \\
\hline Europe & $56(32.0)$ & $50(31.4)$ & $106(31.7)$ \\
\hline Brazil & $35(20.0)$ & $36(22.6)$ & $71(21.3)$ \\
\hline Saudi Arabia & $1(0.6)$ & & $1(0.3)$ \\
\hline \multicolumn{4}{|l|}{ Time from PAH diagnosis months } \\
\hline Mean $\pm S D$ & $26.2 \pm 51.2$ & $25.3 \pm 47.3$ & $25.7 \pm 49.4$ \\
\hline Median (interquartile range) & $11.4(6.0-24.9)$ & $12.2(6.6-28.4)$ & $12.1(6.3-26.0)$ \\
\hline \multicolumn{4}{|l|}{ Aetiology of PAH } \\
\hline Idiopathic & $114(65.1)$ & 99 (62.3) & $213(63.8)$ \\
\hline Familial & $2(1.1)$ & $3(1.9)$ & $5(1.5)$ \\
\hline Connective tissue disease & $45(25.7)$ & $43(27.0)$ & 88 (26.3) \\
\hline Repaired congenital shunts & $11(6.3)$ & $9(5.7)$ & $20(6.0)$ \\
\hline Drugs and toxins & $3(1.7)$ & $5(3.1)$ & $8(2.4)$ \\
\hline \multicolumn{4}{|l|}{ 6-min walk distance $\mathrm{m}$} \\
\hline Mean $\pm \mathrm{SD}$ & $357.6 \pm 73.1$ & $363.1 \pm 78.5$ & $360.3 \pm 75.7$ \\
\hline Median (interquartile range) & $372.0(305-410)$ & $385.0(306-423)$ & $375.5(306-416)$ \\
\hline \multicolumn{4}{|l|}{ WHO functional class } \\
\hline II & $69(39.4)$ & $71(44.7)$ & $140(41.9)$ \\
\hline III & $104(59.4)$ & 88 (55.3) & 192 (57.5) \\
\hline IV & $2(1.1)$ & & $2(0.6)$ \\
\hline \multicolumn{4}{|l|}{ NT-proBNP" pmol.L ${ }^{-1}$} \\
\hline Mean \pm SD & $170.1 \pm 239.4$ & $189.7 \pm 384.0$ & $179.6 \pm 317.2$ \\
\hline Median (interquartile range) & $56.6(21.6-208.5)$ & $54.3(19.4-201.0)$ & $54.9(19.7-202.6)$ \\
\hline \multicolumn{4}{|l|}{ Baseline sildenafil dose mg } \\
\hline Mean \pm SD & $81.1 \pm 45.1$ & $77.8 \pm 48.5$ & $79.6 \pm 46.7$ \\
\hline Median (interquartile range) & $60(60-75)$ & $60(60-60)$ & $60(60-60)$ \\
\hline
\end{tabular}

Data are presented as $\mathrm{n}(\%)$ unless otherwise stated. BMI: body mass index; PAH: pulmonary arterial hypertension; WHO: World Health Organization; NT-proBNP: N-terminal pro-brain natriuretic peptide. \#: placebo, $n=172$; bosentan, $n=157$. ๆ: placebo, $n=117$; bosentan, $n=110$. 
TABLE 2 Primary end-point events in the placebo and bosentan groups

\begin{tabular}{lcc} 
& Placebo & Bosentan \\
\hline Patients n & 175 & 159 \\
Primary end-point event & $90(51.4)$ & $68(42.8)$ \\
Worsening of PAH & $52(29.7)$ & $32(20.1)$ \\
Hospitalisation for PAH or start of $\boldsymbol{i}$.v. prostanoid therapy & $20(11.4)$ & $25(15.7)$ \\
Death & $18(10.3)$ & $10(6.3)$ \\
Atrial septostomy & $0(0.0)$ & $1(0.6)$ \\
Lung transplantation & $0(0.0)$ & $0(0.0)$
\end{tabular}

Data are presented as $\mathrm{n}(\%)$ unless otherwise stated. Events listed are the first confirmed morbidity/ mortality events. PAH: pulmonary arterial hypertension. ": worsening PAH was 1) "moderately worse" or "markedly worse" symptoms and requirement for addition of PAH therapy (initiation of a subcutaneous or inhaled prostanoid, or transfer to open-label bosentan), or 2) "no change" or "mildly worse" symptoms, and requirement for addition of $\mathrm{PAH}$ therapy and deterioration in 6 -min walk distance $>20 \%$ from the previous visit or $>30 \%$ from the baseline visit.

significant $(\mathrm{p}=0.0106)$ (figure 4). There were no differences between the two groups in change in WHO functional class; time to first occurrence of death, hospitalisation for PAH or start of intravenous prostanoid therapy, atrial septostomy or lung transplantation; or time to death from any cause (table 3). NT-proBNP levels increased with placebo and generally remained stable with bosentan over 20 months of treatment, with a difference between the groups in favour of bosentan $(\mathrm{p}=0.0003)$ (supplementary figure 1).

\section{Safety and tolerability}

Of 334 randomised patients, all but one was included in the safety analyses; the excluded patient was randomly assigned to placebo but did not receive the study drug. Patients in the placebo and bosentan groups received double-blind treatment for a mean \pm SD duration of $30.7 \pm 24.31$ and $26.4 \pm 20.99$ months, respectively. During the double-blind phase of the trial, $22(12.6 \%)$ out of 174 patients in the placebo group and 39 (24.5\%) out of 159 patients in the bosentan group permanently discontinued the study drug due to an AE. The most common AEs experienced by patients receiving bosentan were related to the underlying disease (table 4). No new safety signals were observed.

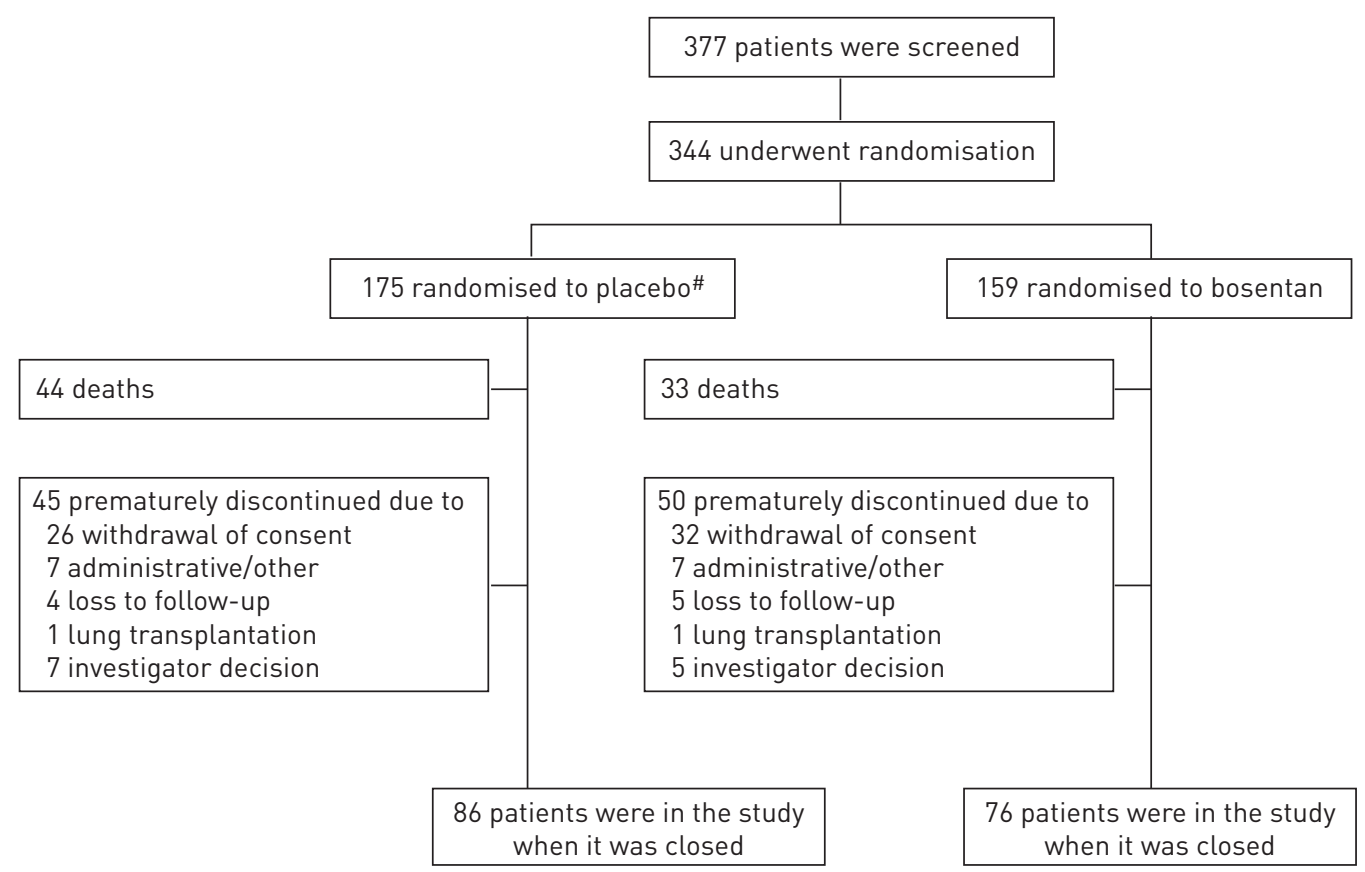

FIGURE 1 Patient disposition. * : one patient randomly assigned to receive placebo did not receive the study drug. 


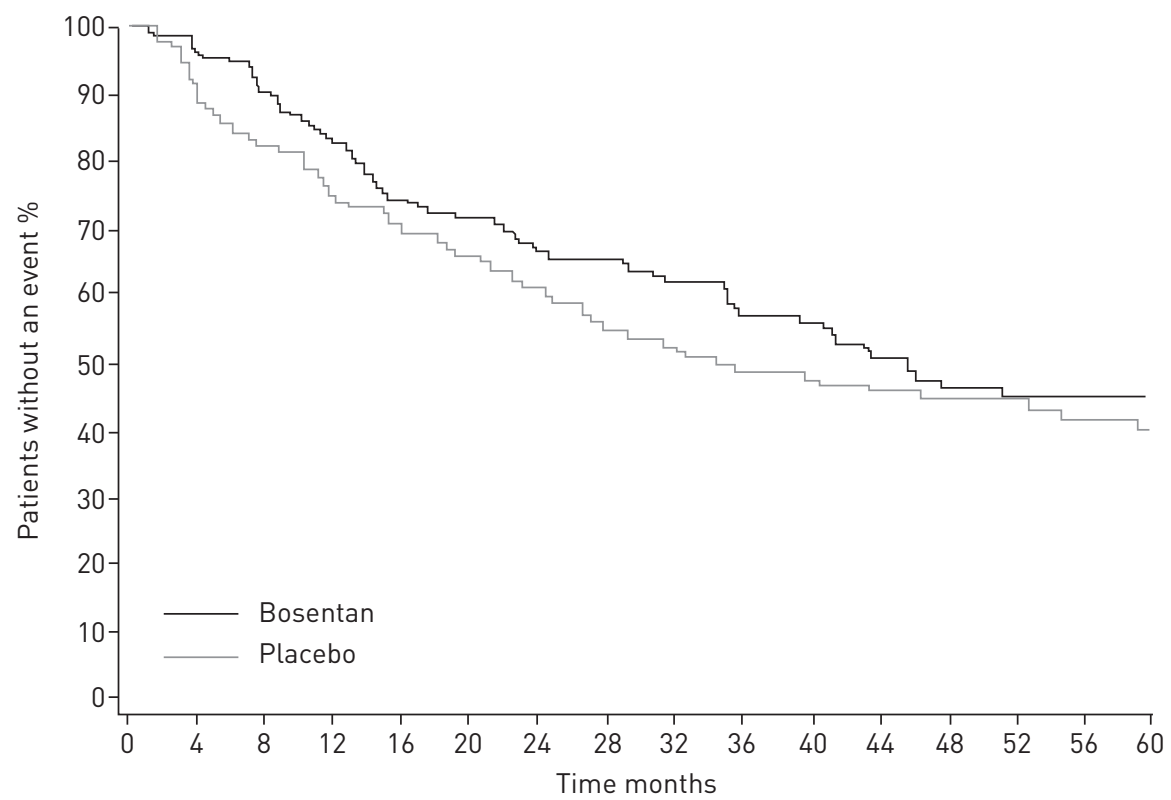

Patients at risk $n$

$\begin{array}{lllllllllllllllll}\text { Placebo } & 175 & 154 & 140 & 123 & 118 & 107 & 90 & 76 & 68 & 61 & 55 & 48 & 43 & 36 & 32 & 26 \\ \text { Bosentan } & 159 & 144 & 128 & 114 & 103 & 97 & 88 & 82 & 69 & 57 & 50 & 42 & 32 & 24 & 21 & 15\end{array}$

FIGURE 2 Time to primary end-point event up to the end of the study. Kaplan-Meier estimates for the time to first primary end-point event (death from any cause, hospitalisation for pulmonary arterial hypertension (PAH) or start of intravenous prostanoid therapy, atrial septostomy, lung transplantation, or worsening PAH) show a treatment effect in favour of bosentan versus placebo (hazard ratio $0.831,97.31 \% \mathrm{Cl} 0.582-1.187 ; \mathrm{p}=0.2508$ by the log-rank test).

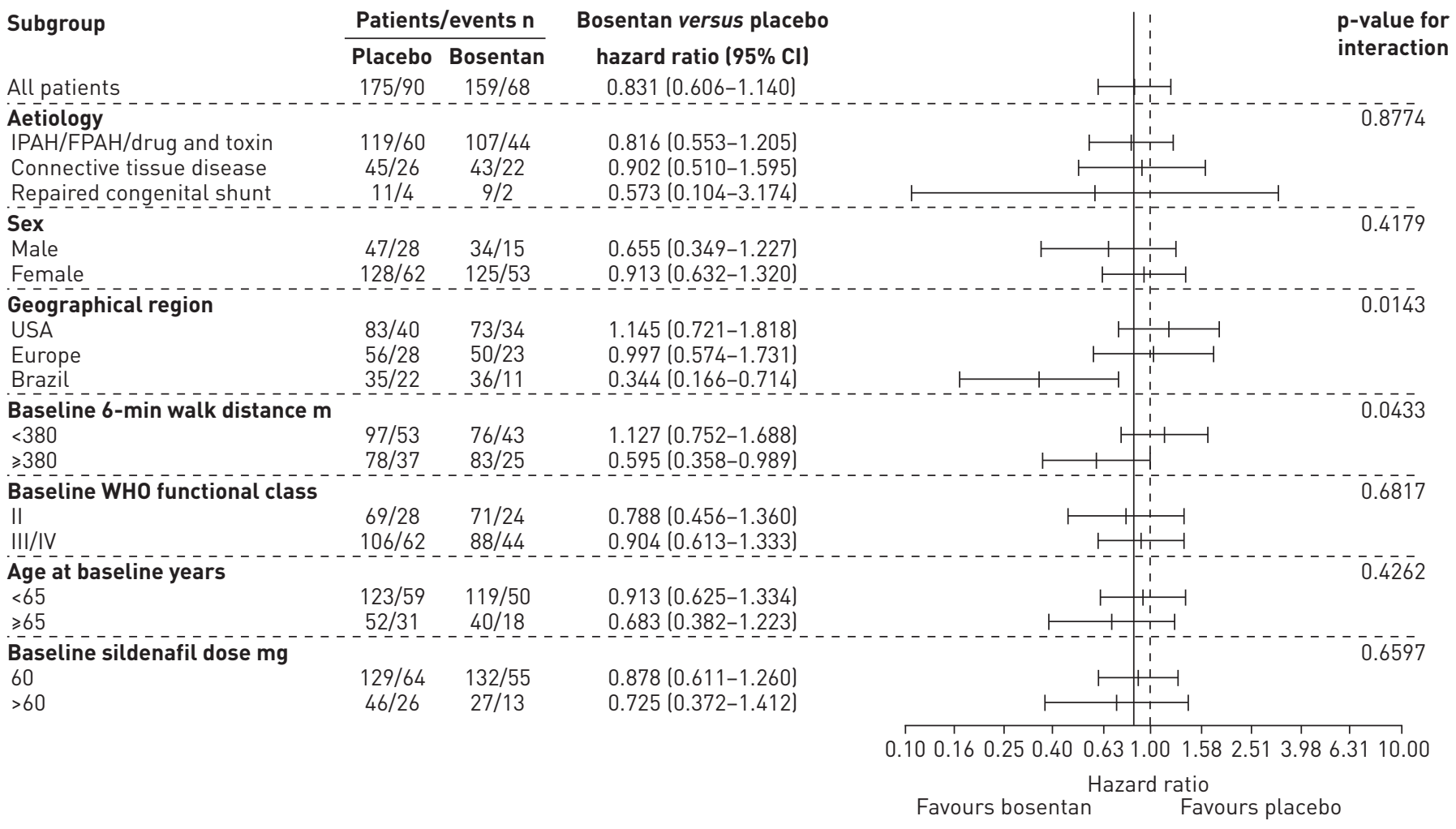

FIGURE 3 Time to primary end-point event by subgroup. For geographical region, one patient from Saudi Arabia was excluded from the analysis. Error bars represent $95 \%$ confidence intervals. IPAH: idiopathic pulmonary arterial hypertension; FPAH: familial pulmonary arterial hypertension; WHO: World Health Organization. 
FIGURE 4 Change from baseline in 6-min walk distance at 16 weeks. The difference between the groups was $21.8 \mathrm{~m} \quad(95 \% \mathrm{Cl} \quad 5.9-37.8 \mathrm{~m}$, $\mathrm{p}=0.0106$ ) by Wilcoxon rank-sum test in favour of bosentan. Data are presented as mean \pm SEM.

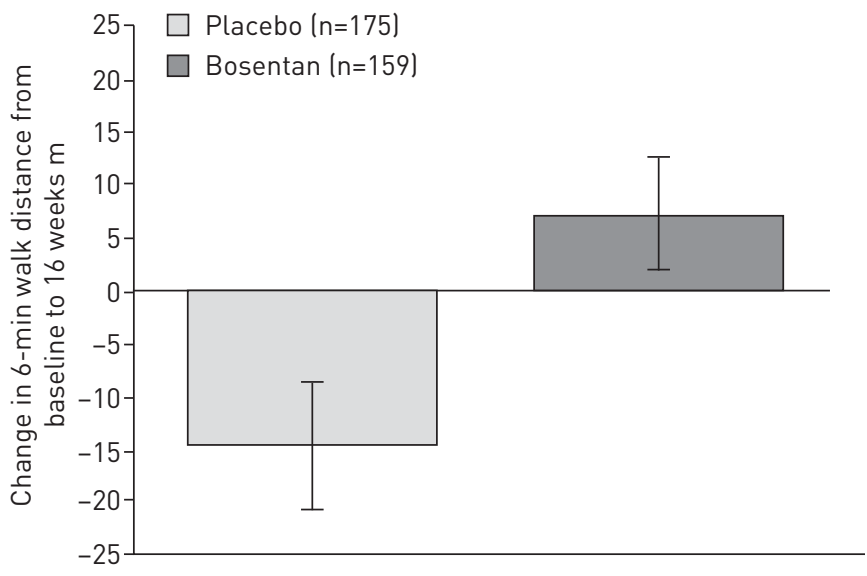

The incidence of ALT or AST more than three times the upper limit of normal on double-blind treatment was $6.4 \%$ in the placebo group $(n=11)$ and $21.8 \%$ in the bosentan group $(n=34)$ (table 4$)$. In the patients treated with bosentan, the majority of the ALT and AST elevations occurred within the first 12 months of treatment (17 (11.4\%) at 4 months and $27(18.9 \%)$ at 12 months). Similar proportions of patients in the placebo and bosentan groups experienced a decrease in haemoglobin levels to $\leqslant 10 \mathrm{~g} \cdot \mathrm{dL}^{-1}(8.8 \%$ and $9.0 \%$, respectively).

\section{Discussion}

The COMPASS-2 study failed to demonstrate a statistically significant effect on the primary endpoint of time to first confirmed morbidity/mortality event, when adding bosentan therapy to sildenafil. There was a nominally significant difference between the treatment groups in favour of bosentan with regards to changes in 6MWD at week 16 and in NT-proBNP; however, no such differences were observed for any other efficacy endpoint. The safety profile of bosentan when added to sildenafil was consistent with the known safety profile of bosentan used as monotherapy. Specifically, the placebo-corrected incidence of liver aminotransferase elevations and haemoglobin decreases are consistent with other long-term bosentan data $[8,9]$, and no new safety signals were observed.

It was assumed that adding bosentan to sildenafil might improve the long-term outcome of PAH patients. This hypothesis was based on the observed effects of bosentan monotherapy on time to clinical worsening in earlier short-term trials [10-12], open-label uncontrolled reports [13] and preliminary data [14] that suggested a clinical benefit when bosentan was combined with sildenafil in patients with PAH.

There are several potential reasons why the COMPASS-2 study did not meet its primary end-point. First, the study was designed to detect a large treatment effect of $>40 \%$; the number of events was too few to allow the detection of smaller, but still potentially meaningful, effects (e.g. 20\%).

\begin{tabular}{|c|c|c|c|}
\hline & Placebo & Bosentan & Treatment effect versus placebo \\
\hline Patients n & 175 & 159 & \\
\hline $\begin{array}{l}\text { Change in WHO functional class from } \\
\text { baseline to } 16 \text { weeks }\end{array}$ & & & $\begin{array}{l}\text { Relative risk of improvement }{ }^{\#} \\
0.98\left(95 \% \mathrm{Cl} 0.60-1.61, p=1.000^{\text {"l }}\right)\end{array}$ \\
\hline Worsened & $17(9.7)$ & 13 (8.2) & \\
\hline Unchanged & $130(74.3)$ & $121(76.1)$ & \\
\hline Improved & $28(16.0)$ & $25(15.7)$ & \\
\hline $\begin{array}{l}\text { Time to death, hospitalisation for PAH, } \\
\text { atrial septostomy or lung } \\
\text { transplantation up to the } \\
\text { end of the study }\end{array}$ & & & $\begin{array}{c}\text { Hazard ratio } 0.963 \\
\left(95 \% \mathrm{Cl} 0.673-1.380, \mathrm{p}=0.8385^{+}\right)\end{array}$ \\
\hline Patients & $67(38.3)$ & $54(34.0)$ & \\
\hline $\begin{array}{l}\text { Time to death from any cause up to the } \\
\text { end of the study }\end{array}$ & & & $\begin{array}{c}\text { Hazard ratio } 0.855 \\
\left(95 \% \mathrm{Cl} 0.544-1.344, \mathrm{p}=0.4974^{+}\right)\end{array}$ \\
\hline Patients & $44(25.1)$ & 33 (20.8) & \\
\hline
\end{tabular}

Data are presented as $\mathrm{n}(\%)$ unless otherwise stated. WHO: World Health Organization; PAH: pulmonary arterial hypertension. "\#: versus no improvement; " : Fisher's exact test; ${ }^{+}$: log-rank test. 
TABLE 4 Exposure, safety and tolerability during double-blind treatment period

\begin{tabular}{|c|c|c|}
\hline & Placebo & Bosentan \\
\hline Patients $n$ & $174^{\#}$ & 159 \\
\hline \multicolumn{3}{|l|}{ Exposure to double-blind study drug months } \\
\hline Mean \pm SD & $30.7 \pm 24.31$ & $26.4 \pm 20.99$ \\
\hline Median (interquartile range) & $23.3(0.3-85.7)$ & $22.7(0.0-86.7)$ \\
\hline \multicolumn{3}{|l|}{ Variable } \\
\hline Patients with adverse events & $159(91.4)$ & $144(90.6)$ \\
\hline Patients with serious adverse events & $102(58.6)$ & $73(45.9)$ \\
\hline $\begin{array}{l}\text { Patients with adverse events leading to discontinuation of } \\
\text { double-blind treatment }\end{array}$ & $22(12.6)$ & $39(24.5)$ \\
\hline \multicolumn{3}{|l|}{ Patients with adverse events } \\
\hline Worsening of pulmonary arterial hypertension & $61(35.1)$ & $39(24.5)$ \\
\hline Oedema, peripheral & $28(16.1)$ & 30 (18.9) \\
\hline Dyspnoea & $26(14.9)$ & $25(15.7)$ \\
\hline Headache & $24(13.8)$ & $24(15.1)$ \\
\hline Upper respiratory tract infection & $30(17.2)$ & $22(13.8)$ \\
\hline Cough & $21(12.1)$ & 22 (13.8) \\
\hline Chest pain & $16(9.2)$ & $22(13.8)$ \\
\hline Diarrhoea & $19(10.9)$ & 19 (11.9) \\
\hline Pneumonia & $13(7.5)$ & 18 (11.3) \\
\hline Bronchitis & $21(12.1)$ & $17(10.7)$ \\
\hline Dizziness & $18(10.3)$ & $17(10.7)$ \\
\hline Anaemia & $12(6.9)$ & $17(10.7)$ \\
\hline Nausea & $22(12.6)$ & $16(10.1)$ \\
\hline Urinary tract infection & $14(8.0)$ & $16(10.1)$ \\
\hline ALT increase & $8(4.6)$ & $16(10.1)$ \\
\hline Back pain & $23(13.2)$ & $15(9.4)$ \\
\hline Arthralgia & 20 (11.5) & $13(8.2)$ \\
\hline Fatigue & $20(11.5)$ & $13(8.2)$ \\
\hline Syncope & $12(6.9)$ & $8(5.0)$ \\
\hline Hypotension & $7(4.0)$ & $0(0)$ \\
\hline \multicolumn{3}{|l|}{ Laboratory abnormality" } \\
\hline ALT or AST >3×ULN & $11(6.4)$ & $34(21.8)$ \\
\hline ALT or $A S T>8 \times U L N$ & $0(0)$ & $8(5.1)$ \\
\hline Haemoglobin $\leqslant 10 \mathrm{~g} \cdot \mathrm{dL}^{-1}$ & $15(8.8)$ & $14(9.0)$ \\
\hline
\end{tabular}

Data are presented as $n(\%)$ unless otherwise stated. ALT: alanine aminotransferase; AST: aspartate aminotransferase; ULN: upper limit of normal. " : one patient randomly assigned to receive placebo did not receive the study drug and was excluded from the safety analyses. ๆ: placebo, $n=171$; bosentan, $n=156$.

Second, the extent of missing information for the primary end-point (calculated at 20\%) as a result of patients discontinuing the study prematurely, without experiencing a primary end-point, was sizeable. The magnitude of missing data further reduced the statistical power and is an important factor when considering the study outcome. Although efforts were made, prior to study closure, to retrospectively collect additional data about these patients, this was not feasible due to patient privacy restrictions.

Third, the worsening PAH component of the primary end-point, which contributed most to the study outcome, differs from that used in recent PAH studies $[5,15,16]$ in that it was determined using the PGSA score as the initial step, with subsequent consideration of 6MWD and the need for additional PAH therapy. Although the PGSA has not been validated in PAH and is associated with some limitations (e.g. it relies on the patient's recognition of worsening PAH symptoms), it was included in an attempt to ensure a patient-centric approach in conjunction with additional objective measures. At that time, validated PAH-specific patient-reported outcome tools were unavailable. Following the third World Symposium on Pulmonary Hypertension, recommendations regarding composite end-points have evolved and this is reflected in the design of more recent $\mathrm{PAH}$ studies, which have employed composite primary end-points with more objective criteria for assessing disease progression $[5,16]$.

Fourth, despite employing comparable inclusion and exclusion criteria to those used in PAH studies at the time of the start of COMPASS-2, the patient population observed in COMPASS-2 was slightly older and the patients had more comorbidities compared with those seen in contemporary PAH trials (key comorbidities are listed in table 3 of the supplementary material) $[11,17,18]$. Baseline characteristics, 
such as older age, comorbidities, concomitant medications and higher BMI, may have affected the response to PAH-specific therapy in those patients and, thus, may have led to a diluted treatment effect. This is suggested by the observed improvement in the primary end-point in certain subgroups, such as the patients from Brazil (figure 3), whose baseline characteristics were more typical of patients enrolled in early PAH studies (e.g. low BMI, fewer comorbidities and younger age), and patients who were able to walk $>380 \mathrm{~m}$ at baseline. Such subgroup effects must be interpreted with caution given the lack of statistical significance in the overall population, the absence of correction for multiple testing and the small number of patients in each subgroup. Nevertheless, this observation suggests that the evolution of inclusion/exclusion criteria in subsequent PAH trials, incorporating more stringent screening steps to minimise the enrolment of patients with confounding factors such as comorbidities, is warranted. The need for careful oversight of enrolled patients has been a key lesson from COMPASS-2 and has affected subsequent long-term event-driven trials in $\mathrm{PAH}[5,16]$.

Fifth, co-administration of bosentan and sildenafil is known to reduce plasma levels of sildenafil and to increase the plasma levels of bosentan [19], and this pharmacokinetic interaction may have contributed to the lack of effect on the primary end-point. However, this remains speculative as plasma levels of both drugs were not measured during this study.

Finally, the trial recruited and followed patients over 7.5 years; the long duration of the trial was mainly due to the slow rate of patient enrolment. During this time, the management and treatment options of patients with PAH changed considerably; such changes may have affected the type of patients enrolled and the retention of patients in the trial.

\section{Conclusion}

COMPASS-2 did not demonstrate that adding bosentan to stable sildenafil therapy was superior to sildenafil monotherapy in delaying the time to the first morbidity/mortality event. Given the limitations of the study, the overall results of the COMPASS-2 study must be interpreted with caution.

\section{References}

1 Humbert M, Sitbon O, Simonneau G. Treatment of pulmonary arterial hypertension. N Engl J Med 2004; 351: $1425-1436$

2 Sitbon O, Morrell N. Pathways in pulmonary arterial hypertension: the future is here. Eur Respir Rev 2012; 21: 321-327.

3 Sitbon O, Simonneau G. Optimal management of severe pulmonary arterial hypertension. Eur Respir Rev 2011; 20: 254-261.

4 Ghofrani HA, Humbert M. The role of combination therapy in managing pulmonary arterial hypertension. Eur Respir Rev 2014; 23: 469-475.

5 Pulido T, Adzerikho I, Channick RN, et al. Macitentan and morbidity and mortality in pulmonary arterial hypertension. N Engl J Med 2013; 369: 809-818.

6 Galiè N, Seeger W, Naeije R, et al. Comparative analysis of clinical trials and evidence-based treatment algorithm in pulmonary arterial hypertension. J Am Coll Cardiol 2004; 43: 81S-88S.

7 Hoeper MM, Oudiz RJ, Peacock A, et al. Endpoints and clinical trial designs in pulmonary arterial hypertension: clinical and regulatory perspectives. J Am Coll Cardiol 2004; 43: 48S-55S.

8 Simonneau G, Galiè N, Jansa P, et al. Long-term results from the EARLY study of bosentan in WHO functional class II pulmonary arterial hypertension patients. Int J Cardiol 2014; 172: 332-339.

9 Actelion Pharmaceuticals UK Ltd. Tracleer (bosentan) $62.5 \mathrm{mg}$ film-coated tablets. www.medicines.org.uk/emc/ medicine/20422/SPC/Tracleer+(bosentan)+62\%205mg+film-coated+tablets/ Date last accessed: September, 2014. Date last updated: January, 2015.

10 Channick RN, Simonneau G, Sitbon O, Effects of the dual endothelin-receptor antagonist bosentan in patients with pulmonary hypertension: a randomised placebo-controlled study. Lancet 2001; 358: 1119-1123.

11 Rubin LJ, Badesch DB, Barst RJ, et al. Bosentan therapy for pulmonary arterial hypertension. N Engl J Med 2002; 346: 896-903.

12 Galiè N, Rubin LJ, Hoeper MM, Treatment of patients with mildly symptomatic pulmonary arterial hypertension with bosentan (EARLY study): a double-blind, randomised controlled trial. Lancet 2008; 371: 2093-2100.

13 Minai OA, Arroliga AC. Long-term results after addition of sildenafil in idiopathic PAH patients on bosentan. South Med J 2006; 99: 880-883.

14 Hoeper MM, Faulenbach C, Golpon H, Combination therapy with bosentan and sildenafil in idiopathic pulmonary arterial hypertension. Eur Respir J 2004; 24: 1007-1010.

15 EU Clinical Trials Register. EudraCT number: 2009-014490-41. www.clinicaltrialsregister.eu/ctr-search/trial/2009014490-41/GB Date last accessed: September, 2014.

16 EU Clinical Trials Register. EudraCT number: 2009-011150-17. www.clinicaltrialsregister.eu/ctr-search/trial/ 2009-011150-17/GB Date last accessed: September, 2014.

17 Humbert M, Barst RJ, Robbins IM, Combination of bosentan with epoprostenol in pulmonary arterial hypertension: BREATHE-2. Eur Respir J 2004; 24: 353-359.

18 Galiè N, Ghofrani HA, Torbicki A, et al. Sildenafil citrate therapy for pulmonary arterial hypertension. $N$ Engl J Med 2005; 353: 2148-2157.

19 Paul GA, Gibbs JS, Boobis AR, Bosentan decreases the plasma concentration of sildenafil when coprescribed in pulmonary hypertension. Br J Clin Pharmacol 2005; 60: 107-112. 